\title{
Colorful surfaces for radiative cooling
}

\author{
Lyu Zhou, ${ }^{\text {a Jacob Rada, }}{ }^{\text {a }}$ Haomin Song, ${ }^{\text {a Boon Ooi, }},{ }^{\text {Z Zongfu Yu, }}$ \\ and Qiaoqiang Gan ${ }^{\mathrm{a}, *}$ \\ ${ }^{a}$ The State University of New York at Buffalo, Department of Electrical Engineering, \\ Buffalo, New York, United States \\ ${ }^{\mathrm{b}}$ King Abdullah University of Science and Technology, Photonics Laboratory, \\ Thuwal, Saudi Arabia \\ ${ }^{c}$ University of Wisconsin-Madison, Department of Electrical and Computer Engineering, \\ Madison, Wisconsin, United States
}

\begin{abstract}
Daytime radiative cooling has attracted extensive research interest due to its potential impact for energy sustainability. To achieve subambient radiative cooling during the daytime, a white surface that strongly scatters incident solar light is normally desired. However, in many practical applications (e.g., roofing materials and car coatings), colored surfaces are more popular. Because of this, there is a strong desire to develop colorful surfaces for radiative cooling. We summarize the general design criteria of radiative cooling materials with different colors and discuss the limitations in cooling performance. Major efforts on this specific topic are reviewed with some suggested topics for future investigation. (C) 2021 Society of Photo-Optical Instrumentation Engineers (SPIE) [DOI: 10.1117/1.JPE.11.042107]
\end{abstract}

Keywords: radiative cooling; color; photonics; thermal management.

Paper 21051MVSS received Jul. 4, 2021; accepted for publication Sep. 22, 2021; published online Oct. 7, 2021.

\section{Introduction}

Cooling is a significant end-use of energy and a major driver of peak electricity demand. According to the U.S. Department of Energy's estimation, air conditioning consumes 15\% of the primary energy used by buildings in the United States. ${ }^{1}$ This percentage of electricity consumption for cooling is much higher in tropical countries (e.g., 70\% in Saudi Arabia ${ }^{2}$ ). In North America areas, most roofs of houses are dark in color, which will introduce significant solar heating to the living environment. Therefore, the roof is an important target to improve the energy efficiency for building and house designs. Another major application of air conditioning is in automobiles. Of the fuel used in vehicles, 29\% is used for cooling and $25 \%$ to $33 \%$ for heat dissipation. ${ }^{3}$ In particular, during the summer, the temperature inside a car parked in direct sunlight can reach $50^{\circ} \mathrm{C}$, which can result in serious injuries or even deaths to humans and pets who are in the vehicle. ${ }^{4}$ Therefore, a passive cooling strategy that cools without electricity could significantly impact global energy consumption, as well as human (especially infant) and pet safety.

Radiative cooling is a promising candidate to meet this cooling need for overheated spaces under direct Sun illumination. ${ }^{5}$ The Earth's atmosphere has a transparency window between 8 and $13 \mu \mathrm{m}$ for electromagnetic waves, corresponding to the peak thermal radiation spectral range of terrestrial objects at typical ambient temperatures (e.g. $\sim 20^{\circ} \mathrm{C}$ to $45^{\circ} \mathrm{C}$ over summertime in tropical areas). This transparency window is a cooling channel for thermal emission through which a thermal body on the Earth's surface can radiate heat into the cold outer space. In the past decade, radiative cooling technologies received emerging interest due to their cooling effect with no consumption of electricity. ${ }^{5-8}$ However, most conventional radiation cooling technologies work at night only since solar heating is dominant during the day. ${ }^{9-15}$ Since the first experimental demonstration of daytime radiative cooling disclosed by Raman et al., ${ }^{16}$ it has emerged as a new energy sustainability research topic for improved heating, ventilation, and air conditioning

*Address all correspondence to Qiaoqiang Gan, qqgan@buffalo.edu 
(HVAC) applications. Experimental daytime radiative cooling strategies were demonstrated with a reported cooling power of $\sim 100 \mathrm{~W} / \mathrm{m}^{2}$ during a sunny day with access to the clear sky ${ }^{17-24} \mathrm{In}$ this mini-review, we discuss the design criteria to integrate radiative cooling functionalities with colorful materials (e.g., roofing materials and paint/coatings of automobiles) and summarize the research progress along this particular research direction. In Sec. 2, we summarize the general design criteria of radiative cooling. In Sec. 3 , we discuss the current colorant products and reveal the potential for further improvement. Then in Secs. 4 and 5, we review the research progress of radiative cooling with colorful surfaces. We conclude this review with a brief perspective for future research.

\section{Criteria of Spectral Selectivity for Radiative Cooling}

The thermal management of outdoor infrastructures, such as building envelopes, is essential to reduce the energy consumption by HVAC applications. Functional cooling roofs ${ }^{25-28}$ and smart windows ${ }^{29-38}$ have been exploited for decades. The major aim is to reduce the heat gain during daytime and cool the space more rapidly after sunset. By manipulating the spectral selectivity of the material, one could effectively control the thermal loads of exterior surfaces. For example, in tropical areas, light-colored roofs are usually preferred to minimize solar absorption [Fig. 1(a)]. With this well-established concept, people developed different types of white cooling roof to improve indoor comfort and save on energy consumed by air conditioning. ${ }^{39,40}$ However, in cooler regions with seasonal conditions, dark roofs are more popular [Fig. 1(b)]. As a result, solar heating is a major thermal load during the summer time. In addition, multiple color coatings are one of the most important options for automobiles to meet personal preferences [Fig. 1(c)], which is less dependent on energy saving or other technical considerations. These colored surfaces will absorb sunlight efficiently and result in a temperature increase when under direct sunlight. Therefore, materials that can simultaneously exhibit colors and lower the surface temperature are highly desired. In past decades, numerous studies were conducted to develop colored paints with minimized solar absorption. ${ }^{41,42}$ However, less attention was paid to the infrared features of these paints. To demonstrate the potential of radiative cooling for these applications, it is necessary to understand the actual optical absorption and thermal emission features of these existing products.

The key to radiative cooling depends on an engineered spectral selectivity. By considering both radiative and non-radiative heat fluxes of an emitter, the net thermal load of an emitter $P_{\text {net }}$ can be calculated by
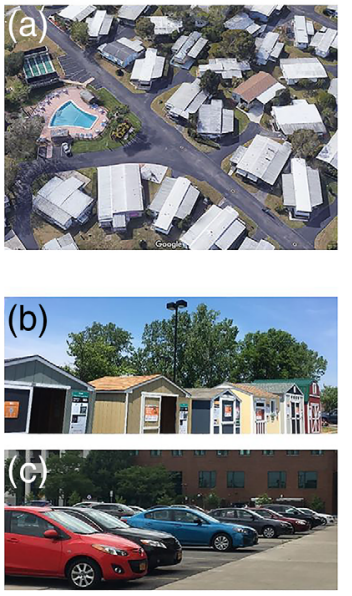

(d)

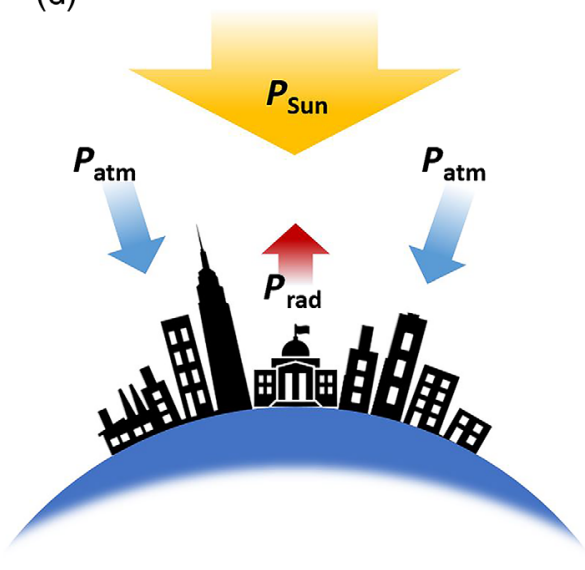

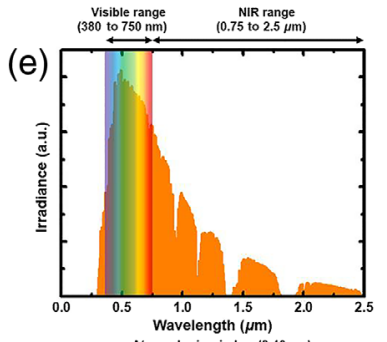

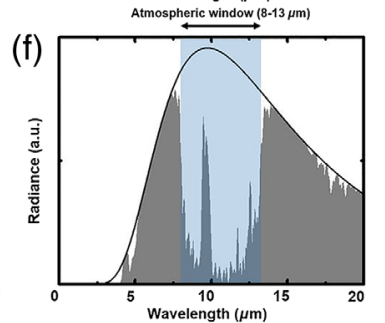

Fig. 1 Spectral selectivity for radiative cooling. (a) Screenshot of a representative community with white roofs (Google map at coordinate: 28.506, -81.411). (b) Photos of colorful roofs and (c) automobiles. (d) Schematic of energy flow of terrestrial objects. (e) Spectral distribution of solar irradiance and (f) atmospheric radiance. 


$$
P_{\text {net }}=P_{\text {rad }}-P_{\text {atm }}-P_{\text {Sun }}-P_{\text {non-rad }}
$$

where $P_{\text {rad }}$ is the outgoing thermal radiation, $P_{\text {atm }}$ is the absorbed atmospheric thermal radiation, $P_{\text {Sun }}$ is the absorbed solar irradiance, and $P_{\text {non-rad }}$ is the non-radiative heat loss. As illustrated in Fig. 1(d), the general criteria are (1) minimizing the absorbed solar irradiance $\left[P_{\text {Sun }}\right.$ in the wavelength range below $2.5 \mu \mathrm{m}$; see Fig. 1(e)] while (2) maximizing the outgoing thermal radiation $\left[P_{\text {rad }}\right.$, especially within the atmospheric transparency window of 8 to $13 \mu \mathrm{m}$; see Fig. 1(f)] and atmospheric thermal radiation $\left(P_{\mathrm{atm}}\right)$. Following these design criteria, considerable research has been reported for daytime radiative cooling. For instance, Hsu et al. ${ }^{43}$ reported an infrared-transparent textile that allows thermal radiation from human skin to transmit through, resulting in colder skin temperature. Zhai et al. ${ }^{44}$ demonstrated a nanoparticle embedded polymer film, which first reported a scalable daytime radiative cooling coating. By introducing hierarchical porous structure into polymer film, Mandal et al. ${ }^{45}$ also reported an efficient daytime radiative cooling material with critical spectra selectivity. However, due to strong scattering and diffraction within solar spectral range, most radiative cooling materials are white in color. ${ }^{46-62}$ For a colored radiative cooling structure, absorption in the visible range is inevitable. Therefore, one needs to consider the balance between solar heating and thermal radiation for colored radiative cooling materials. In the following sections, we will discuss the special design criteria for colored emitters and review the research progress along this particular path.

\section{Commercial Colorful Paints/Coatings}

As a mature market, paints and pigments have been widely used in industrial and civil applications. These commercial products are mixtures of multiple chemicals, ${ }^{63,64}$ which are mostly inexpensive, durable, and scalable, and have been massively deployed in surfaces such as building envelopes, automobile coatings, clothes, and billboards. In recently reported literature for radiative cooling, researchers employed selected commercially available paints as the control to compare with their proposed cooling materials and structures. ${ }^{65-68}$ In order to reveal the potential of colored radiative cooling, we first discuss the spectral characteristics of selected commercial products to reveal the radiative cooling potential.

\subsection{Roofing Materials}

Colorful roofing materials are major solar heating loads to residential houses. Better roofing materials with minimum heating effects are highly desired. The U.S. Environmental Protection Agency predicts an electricity savings of $10 \%$ if colored roofs can be replaced by white ones. ${ }^{69}$ Recently, a roof-cooling project performed by Lawrence Berkeley National Laboratory reported color-matched cool roof tiles with a solar reflectance of over $40 \%$, while showing identical color to regular painted roofs [Fig. 2(a)]. ${ }^{70}$ In particular, the cool roof tiles exhibit over $70 \%$ reflectance in near-infrared (NIR) range. When implemented in tropical or hot-weather areas such as California, the peak temperature of these cool roof tiles is expected to be $\sim 10 \mathrm{~K}$ lower than regular roofs with similar colors, corresponding to the reduction of $\sim 7 \%$ to $15 \%$ in annual cooling power consumption in a regular residential house. ${ }^{70}$ However, in this report, the actual radiative cooling contribution was not revealed. Here we selected six metal roof samples with different colors [see the upper panel in Fig. 2(b), samples from Drexel Metals Inc. ${ }^{71}$ ] to measure their optical and thermal spectra. One can see that all samples show strong thermal emission in the spectral range of 8 to $13 \mu \mathrm{m}$ [Fig. 2(b)]. Therefore, these commercial coatings already meet the requirement for radiative cooling. However, in the visible range, their absorption spectra are mostly broadband, resulting in a strong solar heating effect. Under direct sunlight illumination, their surface temperatures are usually very high (much higher than the ambient temperature).

\subsection{Automobile Coatings}

Another popular color painting is automobile coating. Cooling is highly desired, especially when the air conditioning system of the automobile is not operating in an open parking lot under direct 
(a)
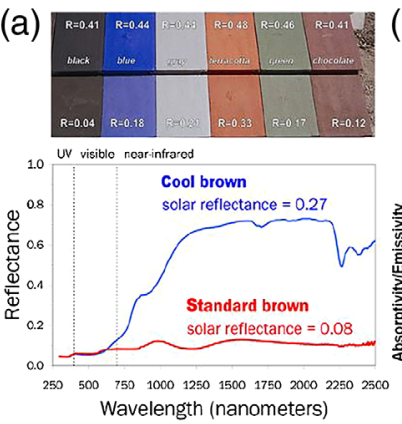

(b)
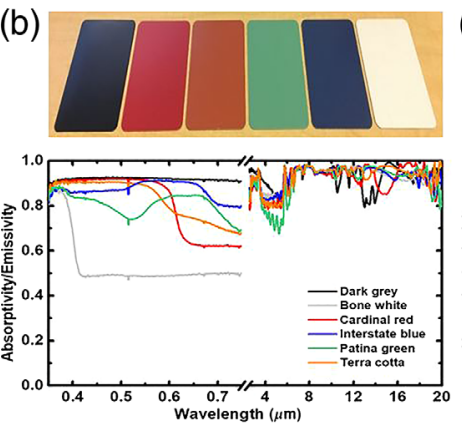

(c)
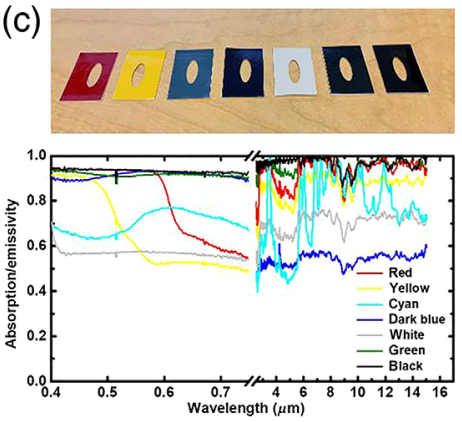

(d)

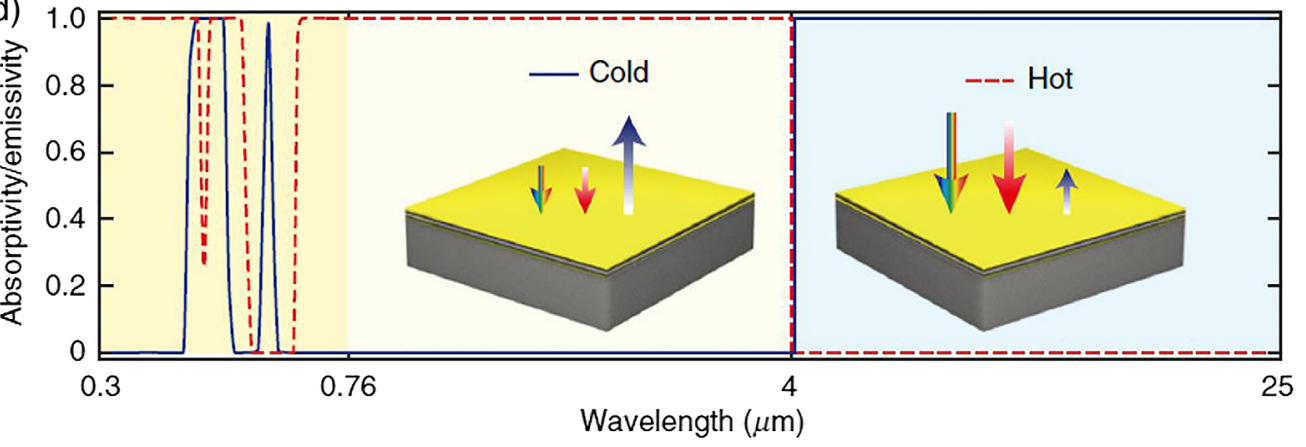

Fig. 2 Spectral features of commercial car paints and roofing materials. (a) Photos and solar spectrum reflectance of the cool roofing materials; (b) photos and absorption spectrum of the metal roofs; (c) photos and absorption spectrum of the Toyota automobile color cards. (d) Spectrum of two surfaces with the same color but different thermal responses. (a) Reprinted with permission from Lawrence Berkeley National Laboratory and (d) reprinted with permission from Springer Nature.

sunlight. Here we selected seven commercial color cards (2001 Toyota/Lexus exterior colors) and measured their optical absorption and thermal emission properties [Fig. 2(c)]. Similar to the colored roofing samples, the automobile coatings show broadband absorption in visible range as well, which intuitively lead to a strong solar heating effect.

To address this solar heating issue, one needs to have better thermal management while preserving the color. By examining the absorbed solar powers in different regions, one can effectively minimize the solar heating while preserving its colorful appearance. The absorbed solar power of a surface can be described by

$$
P_{\text {Sun }}=\int_{0.28 \mu \mathrm{m}}^{0.38 \mu \mathrm{m}} I_{\mathrm{AM} 1.5}(\lambda) \varepsilon(\lambda) \mathrm{d} \lambda+\int_{0.38 \mu \mathrm{m}}^{0.7 \mu \mathrm{m}} I_{\mathrm{AM} 1.5}(\lambda) \varepsilon(\lambda) \mathrm{d} \lambda+\int_{0.7 \mu \mathrm{m}}^{2.5 \mu \mathrm{m}} I_{\mathrm{AM} 1.5}(\lambda) \varepsilon(\lambda) \mathrm{d} \lambda,
$$

where $I_{\mathrm{AM} 1.5}(\lambda)$ represents the solar spectral irradiance at air mass 1.5 , and $\varepsilon(\lambda)$ is the emissivity spectrum of the surface. Considering ultraviolet (UV: 0.28 to $0.38 \mu \mathrm{m})$, visible $(0.38$ to $0.7 \mu \mathrm{m}$ ), and NIR $(0.7$ to $2.5 \mu \mathrm{m})$ regions of the solar spectrum, the irradiance powers in each region correspond to $6.4 \%, 45.5 \%$, and $48.1 \%$ of the total power, respectively. Since the color perception range for human eyes is mainly in the visible range, ${ }^{72}$ the thermal loads of two emitters with identical color can be different under solar illumination. In addition, even for an identical color, different color formation mechanisms can also result in different thermal loads. For instance, $\mathrm{Li}$ et al. ${ }^{66}$ analytically discussed radiative cooling structures for two different situations. As shown in Fig. 2(d), the "cold" emitter should only absorb a narrow band of light in the visible range, resulting in a color that can be described by the cyan, magenta, and yellow colorimetric chart. ${ }^{73}$ It should also reflect other visible and NIR wavelengths to minimize the solar heating effect. On the other hand, in the long-wavelength infrared (LWIR) range, the optimized cold emitter should be strongly emissive to enable radiative cooling. In contrast, the "hot" emitter absorbs a broadband light in the visible range and reflects a relatively narrow band of light to create colors described by the red, green, and blue (RGB) colorimetric chart. As shown by the red dashed line in 
Fig. 2(d), it has high absorption in NIR range, and low emission in LWIR range. Consequently, although these two emitters show similar colors, the thermal response of them under sunlight is drastically different, i.e., the cold emitter can realize a subambient cooling effect while the hot emitter cannot. Following this concept, one could design the colored emitter to obtain optimized cooling performance under different environmental conditions as will be summarized in the next section.

\section{Integration of Radiative Cooling Materials with Existing Colorants}

\subsection{Integration with Porous Polymers}

The colored radiative cooling material inevitably increases solar heating and degrades the cooling power. As a result, subambient cooling is more challenging to realize using colored materials. Instead, the cooling effect of these colored materials is usually compared to their counterparts that have not been engineered with thermal emission in mind. In particular, structures that can dynamically switch between opaque and colored modes offer a better solution for future smart building envelope materials. Mandal et al. ${ }^{74}$ reported porous polymer coatings for dynamic thermal regulation. By wetting the porous polymers with refractive-index-matched fluids, the polymer coatings are able to switch from the scattering mode to the transparent mode, corresponding to a transmittance change by $74 \%$, as shown in Figs. 3(a) and 3(b). In addition, when coupled with a colored background, the coating can dynamically change from white to different colors [Fig. 3(c)], indicating its tunability between cooling and colored modes. More recently, another dynamic switchable coating was also demonstrated by introducing controllable cavitation in PDMS thin films [Fig. 3(d)] ${ }^{75}$ By applying a mechanical force on the surface, the microcavity can be reversibly dilated and sealed, resulting in a transition between opaque and transparent modes. Similar to Ref. 74, this transparent mode was coupled with a colored background to realize coloration.

\subsection{Integration with Pigments}

Integrating pigments into fabric materials has proved effective for colorful radiative cooling, in particular with human body thermal management. Conventional colored fabrics are highly

(a)

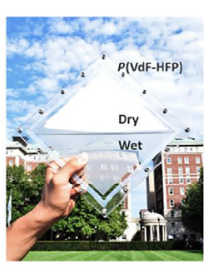

(b)

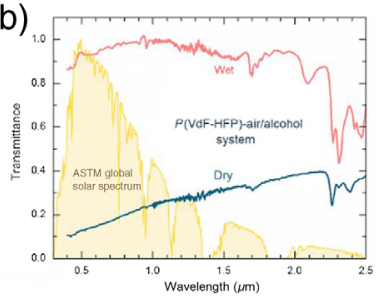

(c)

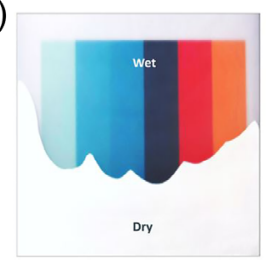

(d)

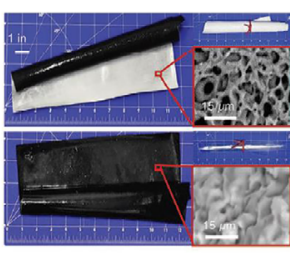

(e)

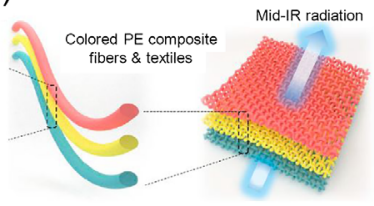

(f)

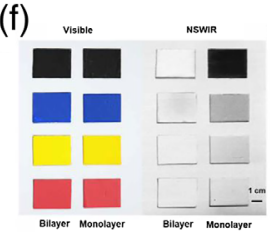

(g)

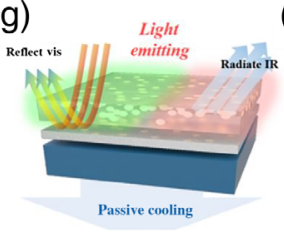

(h)

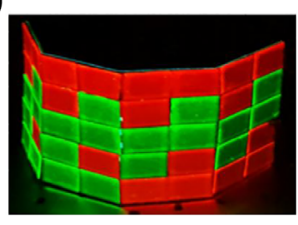

Fig. 3 Colorful radiative cooling integrated with pigments/dyes. (a) Photo with representation of the porous PVDF in dry and wet modes; (b) measured transmittance spectrum of the porous PVDF in wet and dry modes; (c) photos of porous PVDF coupled with colored background. (d) Photos of porous PDMS in opaque mode and transparent mode. (e) Schematic of colored fabric. (f) Photos and infrared images of bilayer colorful coating. (g) Schematic of a photoluminescence emitter for radiative cooling; (h) photo of photoluminescence cooling pixels. (a)-(c) Reprinted with permission from Elsevier B.V., (d) reprinted with permission from Wiley, (e) reprinted with permission from Elsevier B.V., (f) reprinted with permission from American Association for the Advancement of Science, and $(\mathrm{g})$ and $(\mathrm{h})$ reprinted with permission from American Chemical Society. 
absorptive in the infrared spectral region, which hinders the heat dissipation of skin. Cai et al. ${ }^{76}$ provided a solution to modulate both visible and infrared spectra of fabric materials. As shown in Fig. 3(e), by mixing infrared-transparent pigments into polyethylene fabrics, the obtained composite exhibits high transmittance in the infrared range, while maintaining a vivid coloration. Consequently, this textile allows efficient thermal radiation from human skin, therefore reducing the temperature. Although this colored fabric does not emit heat intrinsically, it can be employed as an efficient scattering film to reduce the incident solar irradiance. ${ }^{43,50}$ In addition, direct integration of colored pigments with radiative cooling material was also explored by fully exploiting the penetration depth of light within different wavelength ranges. ${ }^{65}$ As shown in Fig. 3(f), the researchers proposed a bilayer colored painting by coating the NIR reflective substrate (PVDF or $\mathrm{TiO}_{2}$ coating) with a thin, colored layer. As a result, the overall absorption in NIR is reduced significantly when the thickness of the color coating is controlled within tens to hundreds of micrometers. Therefore, a colder temperature can be obtained under direct sunlight illumination compared with a single-layered color paint.

\subsection{Integration with Photoluminescence}

Another method to promote colored radiative cooling is integrating light emitting materials with thermal emission films. ${ }^{77-79}$ Normally, the colored paints or pigments will inevitably result in solar heating. However, light-emitting materials, such as photoluminescent dyes, can partially convert the UV portion of solar irradiance into emitted light, hence slightly reducing the solar-heat conversion. With optimized structures, Jeon et al. ${ }^{78}$ reported colored emitters that can realize subambient cooling performance under $800 \mathrm{~W} / \mathrm{m}^{2}$ solar illumination. Intriguingly, these photoluminescent films can produce vivid colors in dark environments at night [Figs. 3(g) and 3(h)].

In short, this section summarized radiative cooling progresses using colored absorbing materials. To minimize the solar absorption, the emitter needs to be highly reflective in the visible and NIR range. Considering the radiative cooling power of $\sim 100 \mathrm{~W} / \mathrm{m}^{2}$ that can be obtained by white emitters and systems, the optical absorption of the colored materials cannot exceed $10 \%$ of the regular one Sun solar energy $\left(\sim 1000 \mathrm{~W} / \mathrm{m}^{2}\right)$. Therefore, this type of colored radiative cooling materials requires an even more stringent spectral selectivity than white cooling materials. ${ }^{80-90}$ On the other hand, due to this inevitable optical absorption, the cooling power of these colored absorbing surfaces is generally lower than that obtained by white materials. Next, we will discuss another strategy to enable colorful radiative cooling surfaces with structural colors.

\section{Structural Colored Radiative Cooling Materials}

Photonic structural colors received extensive interests in recent decades due to their unique coloration mechanism that is different from conventional pigments. ${ }^{91-100}$ Intriguingly, the color of photonic structures can be designed using engineered subwavelength features to minimize the solar heating effect. For instance, owing to controlled thin film interference (e.g., Fabry-Pérot interference), layered photonic structures can generate designated color within the full visible range. $\mathrm{Li}$ et al. ${ }^{66}$ established a concept of photonic thermal management by fully exploiting the constructive interference to realize cold and hot emitters using different multilayered structures [Fig. 4(a)]. As shown in Fig. 4(b), although both structures have similar absorption features in the visible domain, their absorption in NIR and LWIR ranges are drastically different [Fig. 4(c)]: the total thermal loads of the cold structure and hot structure under one Sun illumination are 716 and $230 \mathrm{~W} / \mathrm{m}^{2}$, respectively. The different thermal loads of these two materials resulted in drastically different temperatures when placed under direct sunlight. Other strategies include silicon nanowire arrays on Ag film [Fig. 4(d)], ${ }^{94}$ metal-insulator-metal cavity structures [Fig. 4(e)], ${ }^{97}$ engineered multilayered films [Fig. 4(f)], ${ }^{98}$ and metallic nanoparticle resonances. ${ }^{100}$ The major target for these works is to realize narrowband optical absorption (i.e., structure-induced colors) and keep the strong thermal emission simultaneously for radiative cooling. However, solarabsorption-induced heating is still inevitable. 
(a)

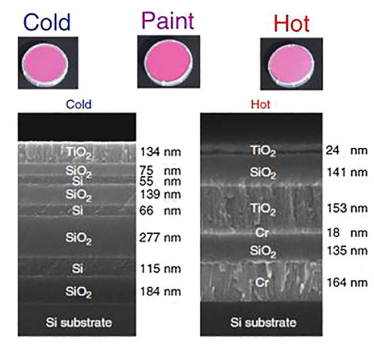

(d)

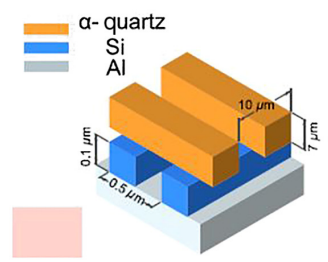

(b)

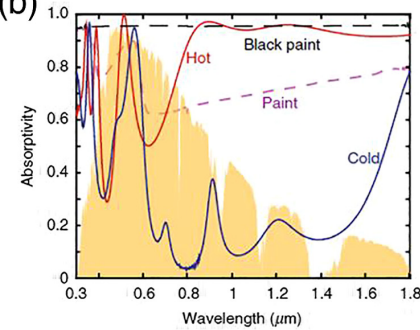

(c)

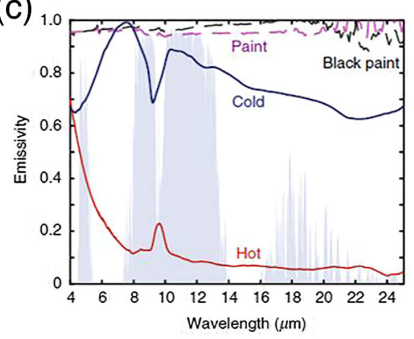

(e)

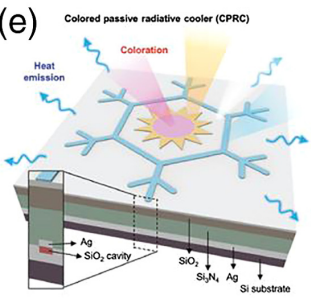

(h) ${ }_{00}$

(g)

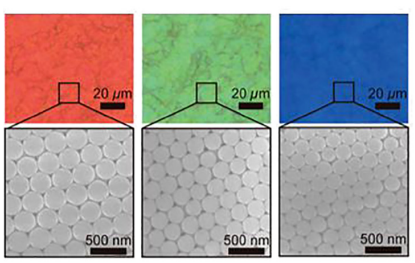

(f)

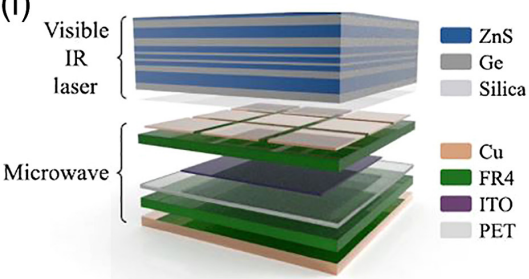

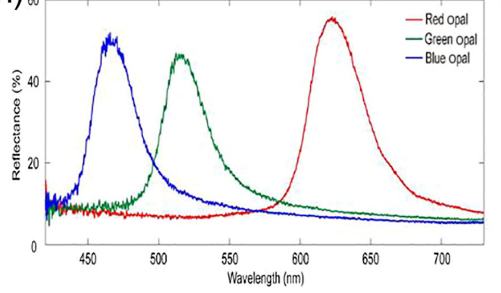

(i)

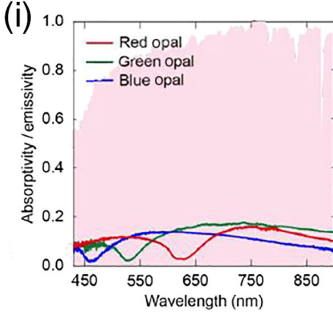

Fig. 4 Structural colored photonic structures. (a) Photos and scanning electron microscopy (SEM) images of cold and hot structures. Measured absorptivity spectra of two structures in (b) solar wavelength range and (c) LWIR range. (d) Schematic of periodic arrays for colored radiative cooler, (e) schematic of a radiative cooler with colorful pattern, and (f) schematic of multispectral camouflage structure. (g) Photos and SEM images of self-assembled nanospheres. (h) Measured reflection and (i) absorption spectra of the silica opal structures. (a)-(c) Reprinted with permission from Springer Nature, (d) reprinted with permission from American Institute of Physics, (e) reprinted with permission from Wiley, (f) reprinted with permission from Springer Nature, and (g)-(i) reprinted with permission from American Chemical Society.

To overcome this challenge, dielectric photonic crystal structures were proposed to minimize the optical absorption. As shown in Fig. 4(g), self-assembled silica opals were coated on silicon wafer by a thin PDMS adhesion layer. By implementing silica opals with different diameters, the close-packed silica photonic crystals can produce different iridescent colors via Bragg diffraction. Importantly, due to the intrinsically low-loss feature of the dielectric materials (e.g., silica spheres), these structural photonic materials can suppress the solar heating effect while preserving the color by reflecting or transmitting narrowband light, making it an ideal candidate for colored radiative cooling. As shown in Fig. 4(h), the silica opal structure can reflect narrowband light, resulting in vivid colors that are described by the RGB color chart. Their optical absorption peaks are all below $10 \%$ in visible range [Fig. 4(i)], which is highly desired by colored radiative cooling. On the other hand, due to the intrinsically strong thermal emission feature of silica nanospheres, the proposed structure obtained intriguing cooling results under regular solar illumination. Therefore, lossless photonic crystal structures combined with thermal emission engineering is one of the promising strategies to realize better radiative cooling performance, which should receive more research efforts for future research and development.

\section{Conclusion and Overview}

In summary, this topic review summarized recent progress in radiative cooling research with colorful surfaces. Two of the most essential criteria, i.e., minimized solar absorption and maximal thermal radiation, are highlighted for optimized cooling performance. Although many 
commercial paints/pigments for roofing materials and automobile coatings already exhibit strong thermal radiation features, their broadband solar absorption introduces a large thermal load to our residential houses and automobiles, resulting in a higher cooling load due to an increase in electricity consumption. Daytime subambient cooling techniques still face the challenge of minimizing the solar heating effects caused by the UV and NIR bands, while preserving a vivid visual color. The implementation of lossless photonic crystal structures offers one of the most promising performance in colorful radiative cooling surfaces, especially when compared to the previously discussed porous films, integrated pigments, or photoluminescent materials. Also we urge researchers to select existing commercial materials as their controls to better reveal the actual value of new radiative cooling materials. Last but not least, large-scale manufacturing processes are essential for the market to actually adopt the new technology to address the emerging global challenges in climate change and energy sustainability.

\section{References}

1. J. K. Kelso, 2011 Buildings Energy Data Book, United States Department of Energy, Washington, DC (2012).

2. A. Demirbas, A. A. Hashem, and A. A. Bakhsh, "The cost analysis of electric power generation in Saudi Arabia," Energy Sources, Part B: Economics, Planning, and Policy 12, 591-596 (2017).

3. S. Chu and A. Majumdar, "Opportunities and challenges for a sustainable energy future," Nature 488, 294-303 (2012).

4. "After six miscarriages, US couple's baby dies in hot car," BBC News, 2019, https://www .bbc.com/news/world-us-canada-49802034 (accessed June 2021).

5. X. Lim, "The super-cool materials that send heat to space," Nature 577, 18-20 (2019).

6. "A heat-radiating material goes sideways to keep its cool," Nature 590, 530 (2021).

7. R. Yang and X. Yin, "Passive cooling in an urban setting," Nat. Sustain. 2, 663-664 (2019).

8. G. Smith and A. Gentle, "Radiative cooling: energy savings from the sky," Nat. Energy 2, 17142 (2017).

9. C. G. Granqvist and A. Hjortsberg, "Surfaces for radiative cooling: silicon monoxide films on aluminum," Appl. Phys. Lett. 36, 139-141 (1980).

10. C. G. Granqvist, "Radiative heating and cooling with spectrally selective surfaces," Appl. Opt. 20, 2606-2615 (1981).

11. C. G. Granqvist, A. Hjortsberg, and T. S. Eriksson, "Radiative cooling to low temperatures with selectivity IR-emitting surfaces," Thin Solid Films 90, 187-190 (1982).

12. S. Catalanotti et al., "The radiative cooling of selective surfaces," Sol. Energy 17, 83-89 (1975).

13. A. K. Head, US patent 3043112A, filed January 12, 1960, and published July 10 (1962).

14. A. R. Gentle and G. B. Smith, "Radiative heat pumping from the Earth using surface phonon resonant nanoparticles," Nano Lett. 10(2), 373-379 (2010).

15. B. Orel, M. K. Gunde, and A. Krainer, "Radiative cooling efficiency of white pigmented paints," Solar Energy 50(6), 477-482 (1993).

16. A. P. Raman et al., "Passive radiative cooling below ambient air temperature under direct sunlight," Nature 515, 540-544 (2014).

17. X. Yin et al., "Terrestrial radiative cooling: using the cold universe as a renewable and sustainable energy source," Science 370, 786-791 (2020).

18. A. R. Gentle and G. B. Smith, "A subambient open roof surface under the mid-summer Sun," Adv. Sci. 2, 1500119 (2015).

19. M. M. Hossain and M. Gu, "Radiative cooling: principles, progress, and potentials," Adv. Sci. 3, 1500360 (2016).

20. Z. Li et al., "Fundamentals, materials, and applications for daytime radiative cooling," Adv. Mater. Technol. 5(5) 1901007 (2020).

21. D. Zhao et al., "Radiative sky cooling: fundamental principles, materials, and applications," Appl. Phys. Rev. 6, 021306 (2019). 
22. M. Zeyghami, D. Y. Goswami, and E. Stefanakos, "A review of clear sky radiative cooling developments and applications in renewable power systems and passive building cooling," Solar Energy Mater. Solar Cells 178, 115-128 (2018).

23. B. Zhao et al., "Radiative cooling: a review of fundamentals, materials, applications, and prospects," Appl. Energy 236, 489-513 (2019).

24. Y. Zhang et al., "Photonics empowered passive radiative cooling," Adv. Photonics Res. 2, 2000106 (2021)

25. X. Li et al., "Multispectral thermal management designs for net-zero energy buildings," ACS Mater. Lett. 2, 1624-1643 (2020).

26. X. Lu et al., "Cooling potential and applications prospects of passive radiative cooling in buildings: the current state-of-the-art," Renew. Sustain. Energy Rev. 65, 1079-1097 (2016).

27. C. Y. Jim, "Air-conditioning energy consumption due to green roofs with different building thermal insulation," Appl. Energy 128, 49-59 (2014).

28. D. Zhao et al., "Roof-integrated radiative air-cooling system to achieve cooler attic for building energy saving," Energy Build. 203, 109453 (2019).

29. R. Mi et al., "Scalable aesthetic transparent wood for energy efficient buildings," Nat. Commun. 11, 3836 (2020).

30. S. Lina et al., "Direct spray-coating of highly robust and transparent $\mathrm{Ag}$ nanowires for energy saving windows," Nano Energy 62, 111-116 (2019).

31. E. L. Runnerstrom et al., "Nanostructured electrochromic smart windows: traditional materials and NIR-selective plasmonic nanocrystals," Chem. Commun. 50, 10555 (2014).

32. G. A. Niklasson and C. G. Granqvist, "Electrochromics for smart windows: thin films of tungsten oxide and nickel oxide, and devices based on these," J. Mater. Chem. 17, 127-156 (2006).

33. D. T. Gillaspie, R. C. Tenent, and A. C. Dillon, "Metal-oxide films for electrochromic applications: present technology and future directions," J. Mater. Chem. 20, 9585-9592 (2010).

34. C. G. Granqvist, "Oxide electrochromics: an introduction to devices and materials," Sol. Energy Mater. Sol. Cells 99, 1-13 (2012).

35. V. K. Thakur et al., "Hybrid materials and polymer electrolytes for electrochromic device applications," Adv. Mater. 24, 4071-4096 (2012).

36. C. G. Granqvist, "Electrochromics for smart windows: oxide-based thin films and devices," Thin Solid Films 564(1), 1-38 (2014).

37. C. Zhou et al., "Transparent bamboo with high radiative cooling targeting energy savings," ACS Mater. Lett. 3, 883-888 (2021).

38. X. H. Li et al., "Broadband light management with thermochromic hydrogel microparticles for smart windows," Joule 3, 290-302 (2019).

39. H. Zhong et al., "Hierarchically hollow microfibers as a scalable and effective thermal insulating cooler for buildings," ACS Nano 15(6), 10076-10083 (2021).

40. T. Li et al., "A radiative cooling structural material," Science 364, 760-763 (2019).

41. G. B. Smith et al., "Coloured paints based on iron oxide and silicon oxide coated flakes of aluminium as the pigment, for energy efficient paint: optical and thermal experiments," Sol. Energy Mater. Sol. Cells 79, 179-197 (2003).

42. G. B. Smith et al., "Coloured paints based on coated flakes of metal as the pigment, for enhanced solar reflectance and cooler interiors: description and theory," Sol. Energy Mater. Sol. Cells 79, 163-177 (2003).

43. P. C. Hsu et al., "Radiative human body cooling by nanoporous polyethylene textile," Science 353, 1019-1023 (2016).

44. Y. Zhai et al., "Scalable-manufactured randomized glass-polymer hybrid metamaterial for daytime radiative cooling," Science 355, 1062-1066 (2017).

45. J. Mandal et al., "Hierarchically porous polymer coatings for highly efficient passive daytime radiative cooling," Science 362, 315-319 (2018).

46. N. N. Shi et al., "Keeping cool: enhanced optical reflection and radiative heat dissipation in Saharan silver ants," Science 349, 298-301 (2015).

47. S. Atiganyanun et al., "Effective radiative cooling by paint-format microsphere-based photonic random media," ACS Photonics 5(4), 1181-1187 (2018). 
48. Y. Peng et al., "Nanoporous polyethylene microfibres for large-scale radiative cooling fabric," Nat. Sustain. 1, 105-112 (2018).

49. P. Yang, C. Chen, and Z. M. Zhang, "A dual-layer structure with record-high solar reflectance for daytime radiative cooling," Solar Energy 169, 316-324 (2018).

50. X. A. Zhang et al., "Dynamic gating of infrared radiation in a textile," Science 363, 619-623 (2019)

51. A. Leroy et al., "High-performance subambient radiative cooling enabled by optically selective and thermally insulating polyethylene aerogel," Sci. Adv. 5, eaat9480 (2019).

52. H. Zhong et al., "Highly solar-reflective structures for daytime radiative cooling under high humidity," ACS Appl. Mater. Interfaces 12(46), 51409-51417 (2020).

53. Q. F. Guan et al., "Nacre-inspired sustainable coatings with remarkable fire-retardant and energy-saving cooling performance," ACS Mater. Lett. 3, 243-248 (2021).

54. K Zhou et al., "Three-dimensional printable nanoporous polymer matrix composites for daytime radiative cooling," Nano Lett. 21(3), 1493-1499 (2021).

55. Y. Chen et al., "Cellulose-based hybrid structural material for radiative cooling," Nano Lett. 21(1), 397-404 (2021).

56. S. Y. Heo et al., "A Janus emitter for passive heat release from enclosures," Sci. Adv. 6, eabb1906 (2020).

57. M. H. Kang et al., "Outdoor-useable, wireless/battery-free patch-type tissue oximeter with radiative cooling," Adv. Sci. 8, 2004885 (2021).

58. W. Gao et al., "Reconfigurable and renewable nano-micro-structured plastics for radiative cooling," Adv. Funct. Mater. 31, 2100535 (2021).

59. D. Chae et al., "Spectrally selective nanoparticle mixture coating for passive daytime radiative cooling," ACS Appl. Mater. Interfaces 13(18) 21119-21126 (2021).

60. W. Huang et al., "Scalable aqueous processing-based passive daytime radiative cooling coatings," Adv. Funct. Mater. 31, 2010334 (2021).

61. Y. Tian et al., "Superhydrophobic and recyclable cellulose-fiber-based composites for highefficiency passive radiative cooling," ACS Appl. Mater. Interfaces, 13(19), 22521-22530 (2021).

62. J. Wu et al., "Robust hierarchical porous PTFE film fabricated via femtosecond laser for self-cleaning passive cooling," Nano Lett., 21(10), 4209-4216 (2021).

63. H. G. Völz, "Pigments, inorganic, 1. General," in Ullmann's Encyclopedia of Industrial Chemistry Ed., Wiley-VCH, Weinheim (2009).

64. H. Mustroph, "Dyes, general survey," in Ullmann's Encyclopedia of Industrial Chemistry Ed., Wiley-VCH Verlag GmbH \& Co., Weinheim (2014).

65. Y. Chen et al., "Colored and paintable bilayer coatings with high solar-infrared reflectance for efficient cooling," Sci. Adv. 6, eaaz5413 (2020).

66. W. Li et al., "Photonic thermal management of coloured objects," Nat. Commun. 9, 4240 (2018)

67. $\mathrm{X}$. Li et al., "Ultrawhite $\mathrm{BaSO}_{4}$ paints and films for remarkable daytime subambient radiative cooling," ACS Appl. Mater. Interfaces 13(18), 21733-21739 (2021).

68. X. Li et al., "Full daytime sub-ambient radiative cooling in commercial-like paints with high figure of merit," Cell Rep. Phys. Sci. 1, 100221 (2020).

69. Energy Star, "Why ENERGY STAR?," https://www.energystar.gov/products/building products/roof_products (accessed June 2021).

70. H. Akbari et al., Cool Color Roofing Materials, p. 73, Lawrence Berkeley National Laboratory (2006).

71. Drexel Metals, "Drexel metals home: 2020 residential color chart brochure," https://www .drexmet.com/color-cards (accessed June 2021).

72. R. W. Hunt, The Reproduction of Colour, 6th ed., pp. 11-12, Wiley, Chichester, UK (2004).

73. U.S. Geological Survey, “CMYK color chart," https://ngmdb.usgs.gov/fgdc_gds/geolsymstd/ fgdc-geolsymcolorchart.pdf (accessed June 2021).

74. J. Mandal et al., "Porous polymers with switchable optical transmittance for optical and thermal regulation," Joule 3, 3088-3099 (2019).

75. H. Zhao et al., "Switchable cavitation in silicone coatings for energy-saving cooling and heating," Adv. Mater. 32, 2000870 (2020). 
76. L. Cai et al., "Temperature regulation in colored infrared-transparent polyethylene textiles," Joule 3, 1478-1486 (2019).

77. S. Son et al., "Colored emitters with silica-embedded perovskite nanocrystals for efficient daytime radiative cooling," Nano Energy 79, 105461 (2021).

78. S. Jeon et al., "Multifunctional daytime radiative cooling devices with simultaneous lightemitting and radiative cooling functional layers," ACS Appl. Mater. Interfaces 12(49), 54763-54772 (2020).

79. R. A. Yalçın et al., "Colored radiative cooling coatings with fluorescence," J. Photonics Energy 11(3) 032104 (2021).

80. L. Zhu, A. P. Raman, and S. Fan, "Radiative cooling of solar absorbers using a visibly transparent photonic crystal thermal blackbody," Proc. Natl. Acad. Sci. U. S. A. 112(40), 12282-12287 (2015).

81. E. Rephaeli, A. Raman, and S. Fan, "Ultrabroadband photonic structures to achieve highperformance daytime radiative cooling," Nano Lett. 13, 1457-1461 (2013).

82. Z. Chen et al., "Radiative cooling to deep sub-freezing temperatures through a 24-h day-night cycle," Nat. Commun 7, 13729 (2016).

83. B. Bhatia et al., "Passive directional sub-ambient daytime radiative cooling," Nat. Commun. 9, 5001 (2018).

84. R. Zhu et al., "Plasmon-enhanced infrared emission approaching the theoretical limit of radiative cooling ability," Nano Lett. 20(10), 6974-6980 (2020).

85. C. C. Tsai et al., "Physical and behavioral adaptations to prevent overheating of the living wings of butterflies," Nat. Commun. 11, 551 (2020).

86. M. Chen et al., "Designing mesoporous photonic structures for high-performance passive daytime radiative cooling," Nano Lett. 21(3) 1412-1418 (2021).

87. L. Zhou et al., "A polydimethylsiloxane-coated metal structure for all-day radiative cooling," Nat. Sustain. 2, 718-724 (2019).

88. L. Zhou et al., "Hybrid concentrated radiative cooling and solar heating in a single system," Cell Rep. Phys. Sci. 2, 100338 (2021).

89. X. Xue et al., "Creating an eco-friendly building coating with smart subambient radiative cooling," Adv. Mater. 32, 1906751 (2020).

90. M. Zhou et al., "Vapor condensation with daytime radiative cooling," Proc. Natl. Acad. Sci. U. S. A. 118(14), e2019292118 (2021).

91. M. A. Kats et al., "Nanometre optical coatings based on strong interference effects in highly absorbing media," Nat. Mater. 12, 20-24 (2013).

92. W. Fan et al., "Iridescence-controlled and flexibly tunable retroreflective structural color film for smart displays," Sci. Adv. 5(8), eaaw8755 (2019).

93. A. Kristensen et al., "Plasmonic colour generation," Nat. Rev. Mater. 2, 16088 (2017).

94. L. Zhu, A. Raman, and S. Fan, "Color-preserving daytime radiative cooling," Appl. Phys. Lett. 103, 223902 (2013).

95. C. Sheng et al., "Colored radiative cooler under optical Tamm resonance," ACS Photonics 6(10), 2545-2552 (2019).

96. E. Blandre et al., "Microstructured surfaces for colored and non-colored sky radiative cooling," Opt. Express 28(20), 29703-29713 (2020).

97. G. J. Lee et al., "Colored, daytime radiative coolers with thin-film resonators for aesthetic purposes," Adv. Opt. Mater. 6, 1800707 (2018).

98. H. Zhu et al., "Multispectral camouflage for infrared, visible, lasers and microwave with radiative cooling," Nat. Commun. 12, 1805 (2021).

99. H. H. Kim, E. Im, and S. Lee, "Colloidal photonic assemblies for colorful radiative cooling," Langmuir 36, 6589-6596 (2020).

100. R. A. Yalçın et al., "Colored radiative cooling coatings with nanoparticles," ACS Photonics 7, 1312-1322 (2020).

Biographies of the authors are not available. 